\title{
Construction sites as an important driver of dengue transmission: implications for disease control
}

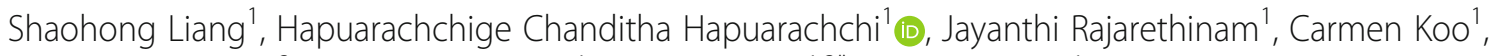
Choon-Siang Tang ${ }^{2}$, Chee-Seng Chong ${ }^{1}$, Lee-Ching $\mathrm{Ng}^{1,3^{*}}$ and Grace Yap ${ }^{1}$

\begin{abstract}
Background: In 2013 and 2014, Singapore experienced its worst dengue outbreak known-to-date. Mosquito breeding in construction sites stood out as a probable risk factor due to its association with major dengue clusters in both years. We, therefore, investigated the contribution of construction sites to dengue transmission in Singapore, highlighting three case studies of large construction site-associated dengue clusters recorded during 2013-16.

Methods: The study included two components; a statistical analysis of cluster records from 2013 to 2016, and case studies of three biggest construction site-associated clusters. We explored the odds of construction site-associated clusters growing into major clusters and determined whether clusters seeded in construction sites demonstrated a higher tendency to expand into major clusters. DENV strains obtained from dengue patients residing in three major clusters were genotyped to determine whether the same strains expanded into the surroundings of construction sites.

Results: Despite less than $5 \%$ of total recorded clusters being construction site-associated, the odds of such clusters expanding into major clusters were 17.4 (2013), 9.2 (2014), 3.3 (2015) and 4.3 (2016) times higher than non-construction site clusters. Aedes premise index and average larvae count per habitat were also higher in construction sites than residential premises during the study period. The majority of cases in clusters associated with construction sites were residents living in the surroundings. Virus genotype data from three case study sites revealed a transmission link between the construction sites and the surrounding residential areas.

Conclusions: Significantly high case burden and the probability of cluster expansion due to virus spill-over into surrounding areas suggested that construction sites play an important role as a driver of sustained dengue transmission. Our results emphasise that the management of construction-site associated dengue clusters should not be limited to the implicated construction sites, but be extended to the surrounding premises to prevent further transmission.
\end{abstract}

Keywords: Dengue, Environmental driver, Construction sites, Genotyping, Surveillance, Control

\section{Background}

Dengue is a complex clinical syndrome with various epidemiological confounders that make disease control a challenging task. Dengue virus (DENV) transmission relies on three obligatory components: host (humans), vector (primarily Aedes aegypti) and the virus (DENV

\footnotetext{
* Correspondence: ng_lee_ching@nea.gov.sg

'Environmental Health Institute, 11, Biopolis Way, \#06-05-08, Singapore

138667, Singapore

${ }^{3}$ School of Biological Sciences, Nanyang Technological University, 60

Nanyang Drive, Singapore 637551, Singapore

Full list of author information is available at the end of the article
}

serotypes 1, 2, 3 and 4). Approximately 3.6 billion people are at risk of DENV infection, with an estimated 50-200 million infections occurring annually [1]. The large-scale re-emergence of dengue fever over the past few decades has raised a serious international public health concern, especially in the tropics and subtropics [2].

Located in the Asia-Pacific region, Singapore is not spared from dengue fever outbreaks despite vector control efforts since 1960s [3, 4]. In 2013 and 2014, the country experienced its worst dengue outbreak known-to-date, with 22,077 (seven deaths) and 18,318 confirmed cases

(c) The Author(s). 2018 Open Access This article is distributed under the terms of the Creative Commons Attribution 4.0 International License (http://creativecommons.org/licenses/by/4.0/), which permits unrestricted use, distribution, and 
(five deaths), respectively [5]. Several epidemiological, entomological and virological parameters have been analysed to determine the possible factors responsible for outbreaks [5-7]. Among many contributors, environmental factors appear to be one of the important influential components that can impact both human and vector populations [8]. Some of these environmental factors can be modified or manipulated to mitigate dengue transmission.

One of such potential environmental factors identified in Singapore is the condition of construction sites. Being an urban city with high population density $(>7000$ people per metre square), Singapore undergoes regular upgrading of its infrastructure. In 2013, more than 2500 construction sites of different scales were recorded. Construction projects primarily rely on foreign labour, including workers from dengue non-endemic or newly endemic countries, who may be immunologically naïve to dengue. Due to the nature of construction work, these workers are unlikely to be permanently stationed, and tend to work at different sites across the country. As construction sites are dynamic environments, different phases of construction may allow the sites to be conducive for Aedes breeding when potential habitats are constantly created or not removed. For example, water puddles on various surfaces, such as concrete floors in uncompleted buildings, are common and have shown to be attractive breeding habitats for Ae. aegypti $[9,10]$. It is highly challenging to manoeuvre among construction materials to properly survey and eliminate all potential breeding habitats. During 2013-15, several major dengue clusters were linked to construction sites [11-13]. Anecdotal construction site outbreaks in Singapore have been reported.

In the present study, we aimed to investigate the contribution of construction sites as an important environmental factor of dengue transmission in Singapore, and further examined this phenomenon through three case studies of large construction site-associated dengue clusters recorded during 2013-16.

\section{Methods}

Exploring the impact of construction site-associated cases on the cluster size

In Singapore, dengue cases are clustered for targeted vector control operational purposes based on their proximity and onset dates. All cases are laboratory-confirmed. A cluster is formed when two cases are located within $150 \mathrm{~m}$ apart from each other and their onset dates are within 14 days. Subsequent cases that fulfil the same criteria are also tagged to the same cluster. Clusters are further stratified into major and minor - a major cluster is defined as having 10 or more reported cases. All cases that do not belong to any cluster are defined as sporadic. When cases occur in a construction site within the boundary of a defined cluster, it is considered as a construction site-associated cluster. In the present study, we analysed the epidemiological records of dengue cases from 2013 to 2016. Clusters were categorised into construction site-associated and non-construction site-associated clusters. We explored the odds of construction site-associated clusters growing into major clusters, the average number of cases per large construction site-associated cluster, and determined whether clusters initiated by construction site-associated cases demonstrated a higher tendency to expand into major clusters. A cluster initiated by construction site-associated cases was defined as having any of the first notified cases being a worker from the construction site. The Odds ratio (OR) with 95\% confidence interval was calculated according to Altman [14].

\section{Case studies: Bedok reservoir road, Choa Chu Kang and Tampines clusters}

Bedok Reservoir Road (BR) and Tampines are located in the eastern region of Singapore, while Choa Chu Kang $(\mathrm{CCK})$ is located in the western region, approximately $21 \mathrm{~km}$ apart. BR is a residential area of $2.4 \mathrm{~km}^{2}$ with 23,600 residents. CCK is a residential area of $6.1 \mathrm{~km}^{2}$ with 174,320 residents. There were 261,240 residents in Tampines within an area of $21 \mathrm{~km}^{2}$. All three clusters were classified operationally as construction site-associated clusters. BR cluster was active from EW35 to 47 in 2013 and recorded 158 cases. CCK cluster recorded 534 cases from EW24 to EW33 in 2014. Tampines cluster recorded 280 cases from EW42 of 2015 to EW6 of 2016. Analysing the epidemiological aspects of clusters, we attempted to find out their similarities and how they may have contributed to the establishment of major clusters. Additionally, DENV strains obtained from dengue patients among construction workers and residents living in BR, CCK and Tampines clusters were genotyped. Genotyping was carried out based on the phylogenetic analysis of envelope $(E)$ gene sequences of DENV as described elsewhere [15].

\section{Results \\ Overall case burden of construction site-associated clusters was significantly high}

A summary of the cluster categories, distribution and case counts is given in Table 1. A cluster was defined as a congregation of two or more cases located within $150 \mathrm{~m}$ apart from each other in 14 days of onset from the last reported case. When cases occurred in a construction site within the boundary of a defined cluster, the cluster was operationally classified as a construction site-associated cluster. Of the 6568 clusters reported from 2013 to 2016, the number of major clusters (each consisting of 10 or more notified cases) ranged between $102(5.9 \%, 2016)$ and $199(11.7 \%, 2013)$. The highest number of major clusters in 2013 was due to the epidemic transmission [5]. Nevertheless, major clusters contributed 
Table 1 Case details of major ( $\geq 10$ cases) and minor ( $<10$ cases) clusters: Overall and construction site-associated clusters

\begin{tabular}{|c|c|c|c|c|c|c|c|c|c|c|c|c|c|c|c|c|}
\hline & \multicolumn{4}{|l|}{2013} & \multicolumn{4}{|l|}{2014} & \multicolumn{4}{|l|}{2015} & \multicolumn{4}{|c|}{2016} \\
\hline Total no. of clusters & \multicolumn{4}{|l|}{1705} & \multicolumn{4}{|l|}{1823} & \multicolumn{4}{|l|}{1319} & \multicolumn{4}{|l|}{1721} \\
\hline \multirow[t]{2}{*}{ No. of clusters based on size ${ }^{a}$} & \multicolumn{2}{|l|}{$\geq 10$} & \multicolumn{2}{|l|}{$<10$} & \multicolumn{2}{|l|}{$\geq 10$} & \multicolumn{2}{|l|}{$<10$} & \multicolumn{2}{|l|}{$\geq 10$} & \multicolumn{2}{|l|}{$<10$} & \multicolumn{2}{|l|}{$\geq 10$} & \multicolumn{2}{|l|}{$<10$} \\
\hline & \multicolumn{2}{|c|}{$199(11.7 \%)$} & \multicolumn{2}{|c|}{$1506(88.3 \%)$} & \multicolumn{2}{|c|}{$133(7.3 \%)$} & \multicolumn{2}{|c|}{$1690(92.7 \%)$} & \multicolumn{2}{|c|}{$108(8.2 \%)$} & \multicolumn{2}{|c|}{$1211(91.8 \%)$} & \multicolumn{2}{|c|}{$102(5.9 \%)$} & \multicolumn{2}{|c|}{1619 (94.1\%, } \\
\hline Average cases per cluster & \multicolumn{2}{|l|}{30.7} & \multicolumn{2}{|l|}{3.3} & \multicolumn{2}{|l|}{39.7} & \multicolumn{2}{|l|}{2.9} & \multicolumn{2}{|l|}{31.3} & \multicolumn{2}{|l|}{2.9} & \multicolumn{2}{|l|}{26.4} & \multicolumn{2}{|l|}{3.0} \\
\hline \multirow[t]{2}{*}{ Construction site-associated clusters } & \multicolumn{2}{|l|}{ Yes } & No & & Yes & & No & & Yes & & No & & Yes & & No & \\
\hline & $50(2$ & & 1655 & $97.1 \%)$ & $88(4$. & 8\%) & 1735 & $95.2 \%)$ & $41(3$ & $.1 \%)$ & 1278 & $96.9 \%)$ & $50(2$ & $.9 \%)$ & 1671 & $97.1 \%$ \\
\hline Cluster size $^{a}$ & $\geq 10$ & $<10$ & $\geq 10$ & $<10$ & $\geq 10$ & $<10$ & $\geq 10$ & $<10$ & $\geq 10$ & $<10$ & $\geq 10$ & $<10$ & $\geq 10$ & $<10$ & $\geq 10$ & $<10$ \\
\hline No. of clusters & 33 & 17 & 166 & 1489 & 32 & 56 & 101 & 1634 & 9 & 32 & 99 & 1179 & 10 & 40 & 92 & 1579 \\
\hline No. of cases & 1336 & 62 & 4777 & 4893 & 2473 & 216 & 2805 & 4707 & 537 & 102 & 2845 & 3391 & 360 & 135 & 2331 & 4661 \\
\hline Average cases per cluster & 40.5 & 3.6 & 28.7 & 3.3 & 77.1 & 3.9 & 27.8 & 2.9 & 59.7 & 3.2 & 28.7 & 2.9 & 36 & 3.4 & 25.3 & 3.0 \\
\hline Odds ratio ${ }^{\mathrm{b}}(95 \% \mathrm{Cl})$ & 17.4( & $9.49-3$ & & & 9.24( & $5.73-1$ & 4.9) & & 3.35 & $(1.55-7$ & .21) & & 4.29 & $(2.07-\varepsilon$ & 3.85) & \\
\hline
\end{tabular}

${ }^{a} \geq 10=$ major clusters; $<10=$ minor clusters

${ }^{\mathrm{b}}$ Odds ratio of construction site-associated clusters expanding into major clusters. $\mathrm{Cl}$ confidence interval

to approximately half of the "clustered" cases in each year, except for 2016 (35.9\%). This highlights the impact of major clusters on the overall dengue case burden in Singapore.

The construction-site associated major clusters recorded 1.41 (2013) - 2.77 (2014) times more cases per cluster than those that were not associated with construction sites. The overall case burden of construction site-associated clusters was significantly higher than that of remaining clusters ( $p$-value $<0.001$ for all 4 years).

\section{The probability of construction site-associated clusters expanding into major clusters was significantly high regardless of the origin of the initial case}

Of all clusters reported from 2013 to 2016, the proportion of construction site-associated clusters was low and ranged from 2.9\% (2013 and 2016) to 4.8\% (2014) (Table 1). However, a substantially high proportion of construction site-associated clusters was major clusters (range: $20 \%$ in $2016-66 \%$ in 2013). The OR of construction site-associated clusters expanding into major clusters was significantly higher than the remaining clusters in each year $(p$-value $<0.01)$ (Table 1$)$.

It was then, determined whether there was any relationship between the size of construction site-associated clusters and the origin of the first notified case (either a worker within the construction sites or a resident living in the vicinity of the construction sites), based on disease onset dates. The findings showed that the probability of expansion of a particular cluster into a major cluster was not dependent on the first notified case being a worker (Table 2). The OR of construction site-initiated clusters (the first case was a worker) expanding into major clusters was not significantly different from those that had residents as the first notified case (Table 2). The proportion of major clusters and the average number of cases per cluster was comparable between the construction site-initiated clusters and those initiated by residents in the vicinity (Table 2).

We, next, investigated whether the scale of a particular construction site has any impact on the final size of construction site-initiated clusters. We used the estimated cost of respective construction projects as a proxy for their scale and classified sites costing 10 million Singapore dollars and above as large-scale projects. The OR of clusters initiated within large-scale construction sites expanding into major clusters was not statistically significant in each year (Table 3).

\section{Virus exchange between the construction sites and surrounding residential areas is likely to drive the rapid expansion of clusters}

We further examined the contribution of construction sites as an important driver of dengue transmission through three case studies of construction site-associated major dengue clusters; Bedok Reservoir Road (BR) in 2013 (Fig. 1), Choa Chu Kang (CCK) in 2014 (Fig. 2) and Tampines during 2015-16 (Fig. 3). These study sites were chosen based on the case burden. Each site recorded the highest number of cases among construction site associated clusters in respective years. The index cases of BR and Tampines clusters were recorded in respective construction sites, whereas that of the CCK cluster was a resident in the neighbouring area. Of 158 cases recorded in the BR cluster, $20.3 \%$ of cases were workers from a construction site located within the cluster boundary. Similarly, $36.3 \%$ and $27.1 \%$ of cases in CCK $(n=534)$ and Tampines $(n=280)$ clusters respectively were among site workers. The spatio-temporal case density analysis showed that the transmission was persistent within each construction site and gradually intensified during the active period of respective clusters (Figs. 1, 2 and 3). We compared the 
Table 2 Summary of construction site-associated clusters in which the first known case was notified from the construction site or from the surrounding areas

\begin{tabular}{|c|c|c|c|c|c|c|c|c|c|c|c|c|c|c|c|c|}
\hline & \multicolumn{4}{|l|}{2013} & \multicolumn{4}{|l|}{2014} & \multicolumn{4}{|l|}{2015} & \multicolumn{4}{|l|}{2016} \\
\hline Total no. of clusters & 50 & & & & 88 & & & & 41 & & & & 50 & & & \\
\hline \multirow{2}{*}{$\begin{array}{l}\text { No. of clusters that notified the } \\
\text { first case from a construction site }\end{array}$} & \multicolumn{2}{|l|}{ Yes } & \multicolumn{2}{|l|}{ No } & \multicolumn{2}{|l|}{ Yes } & \multicolumn{2}{|l|}{ No } & \multicolumn{2}{|l|}{ Yes } & \multicolumn{2}{|l|}{ No } & \multicolumn{2}{|l|}{ Yes } & \multicolumn{2}{|l|}{ No } \\
\hline & \multicolumn{2}{|l|}{35} & \multicolumn{2}{|l|}{12} & \multicolumn{2}{|l|}{54} & \multicolumn{2}{|l|}{28} & \multicolumn{2}{|l|}{25} & \multicolumn{2}{|l|}{12} & \multicolumn{2}{|l|}{31} & \multicolumn{2}{|l|}{9} \\
\hline Cluster size ${ }^{b}$ & $\geq 10$ & $<10$ & $\geq 10$ & $<10$ & $\geq 10$ & $<10$ & $\geq 10$ & $<10$ & $\geq 10$ & $<10$ & $\geq 10$ & $<10$ & $\geq 10$ & $<10$ & $\geq 10$ & $<10$ \\
\hline No. of clusters & 24 & 11 & 9 & 3 & 19 & 35 & 11 & 17 & 6 & 19 & 3 & 9 & 6 & 25 & 3 & 6 \\
\hline No. of cases & 1038 & 44 & 298 & 10 & 1546 & 112 & 836 & 54 & 425 & 59 & 112 & 30 & 231 & 79 & 107 & 25 \\
\hline Average cases per cluster & 43.3 & 4 & 33.1 & 3.3 & 81.4 & 3.2 & 76.4 & 3.2 & 70.8 & 3.1 & 37.3 & 3.3 & 38.5 & 3.2 & 35.7 & 4.2 \\
\hline Odds ratio ${ }^{c}(95 \%$ Cl) & \multicolumn{4}{|c|}{$0.73(0.16-3.22)$} & \multicolumn{4}{|c|}{$0.84(0.33-2.15)$} & \multicolumn{4}{|c|}{$0.95(0.20-4.68)$} & \multicolumn{4}{|c|}{$2.08(0.4-10.8)$} \\
\hline
\end{tabular}

${ }^{a}$ Numbers given here are less than the total number of clusters stated because of the non-availability of index case information from certain clusters

$b^{b} \geq 10=$ major clusters; $<10=$ minor clusters

'Odds ratio of construction site-associated clusters expanding into major clusters. $\mathrm{Cl}=$ confidence interval

envelope gene $(E)$ identity of virus strains $(n=111)$ obtained from construction workers and residents in each study site (BR; $n=35$, CCK; $n=68$ and Tampines; $n=8$ ) to determine whether the expansion of respective clusters was due to spilling over of similar virus strains into surrounding areas.

Of 35 viruses analysed in BR cluster, DENV-1 $(n=32)$ was the most common serotype. The remaining three were DENV-3 viruses. Amongst the DENV-1 strains, 31 viruses (96.9\%) belonged to DENV-1 genotype III, which was the dominant lineage during the 2013-2014 epidemic [5]. The remaining sequence belonged to DENV-1 genotype I. DENV-1 genotype III strains were detected throughout the entire transmission period of BR cluster (Epidemiological week (EW) 35-47), indicating their consistent presence in the cluster (Fig. 4). Moreover, those 31 DENV-1 genotype III strains shared high nucleotide (99.4-100\%) and amino acid (99.3-100\%) similarities. Of them, $22(71 \%)$ sequences belonged to the same variant (13.03) [7]. Interestingly, virus diversity increased during the late phase of the active period of BR cluster (EW4446), during which DENV-3 was also detected, indicating multiple introduction events towards the end of transmission period (Fig. 4).

All virus strains $(n=68)$ genotyped in CCK cluster belonged to DENV-1. Among them, 67 (98.5\%) strains were of genotype III and the remaining strain belonged to genotype I. Likewise in BR cluster, DENV-1 genotype III strains in CCK cluster were closely related (nucleotide similarity of $99.5-100 \%$ and amino acid similarity of 99.1-100\%) throughout the entire period of the cluster (Fig. 5). Sixty-six (98.5\%) sequences of DENV-1 genotype III belonged to the same variant (13.12) [7].

On the other hand, a mixed serotype pattern was observed in the Tampines cluster (Fig. 6). Of eight viruses genotyped in the cluster, five $(62.5 \%)$ belonged to DENV-2 cosmopolitan genotype (clade Ib; $[5,7]$ ) and the remaining viruses belonged to DENV-1 genotype III. Nevertheless, all DENV-2 and DENV-1 virus sequences were closely related and clustered within the same variants of respective serotypes. Four out of five DENV-2 and two out of three DENV-1 viruses shared identical $E$ gene sequences.

In summary, the virus populations in all three study sites were highly homogenous. The dominant strains were introduced at the beginning of the transmission period and circulated throughout the active period of each cluster. These observations indicated that the transmission has primarily been driven by a single introduction event at each study site and the expansion of clusters resulted from spill-over transmission in surrounding areas. Our previous virus genotype data has suggested the exchange of identical virus strains between distant construction sites managed by the same construction company

Table 3 Summary of construction site-initiated ${ }^{a}$ clusters based on the scale of construction projects ${ }^{b}$

\begin{tabular}{|c|c|c|c|c|c|c|c|c|c|c|c|c|c|c|c|c|}
\hline \multirow[b]{2}{*}{ Scale of construction site } & \multicolumn{4}{|l|}{2013} & \multicolumn{4}{|l|}{2014} & \multicolumn{4}{|l|}{2015} & \multicolumn{4}{|l|}{2016} \\
\hline & \multicolumn{2}{|c|}{ Large } & \multicolumn{2}{|l|}{ Small } & \multicolumn{2}{|l|}{ Large } & \multicolumn{2}{|l|}{ Small } & \multicolumn{2}{|l|}{ Large } & \multicolumn{2}{|l|}{ Small } & \multicolumn{2}{|l|}{ Large } & \multicolumn{2}{|l|}{ Small } \\
\hline & \multicolumn{2}{|l|}{12} & \multicolumn{2}{|l|}{18} & \multicolumn{2}{|l|}{15} & \multicolumn{2}{|l|}{40} & \multicolumn{2}{|l|}{13} & \multicolumn{2}{|l|}{18} & \multicolumn{2}{|l|}{17} & \multicolumn{2}{|l|}{11} \\
\hline Cluster size $^{a}$ & $\geq 10$ & $<10$ & $\geq 10$ & $<10$ & $\geq 10$ & $<10$ & $\geq 10$ & $<10$ & $\geq 10$ & $<10$ & $\geq 10$ & $<10$ & $\geq 10$ & $<10$ & $\geq 10$ & $<10$ \\
\hline No. of clusters & 9 & 3 & 12 & 6 & 6 & 9 & 16 & 24 & 2 & 11 & 4 & 14 & 4 & 13 & 4 & 7 \\
\hline Odds ratio ${ }^{b}(95 \%$ Cl) & \multicolumn{4}{|c|}{$1.5(0.29-7.68)$} & \multicolumn{4}{|c|}{$1.0(0.30-3.36)$} & \multicolumn{4}{|c|}{$0.64(0.10-4.14)$} & \multicolumn{4}{|c|}{$0.54(0.10-2.84)$} \\
\hline
\end{tabular}

${ }^{a}$ Construction site-initiated clusters are those that notified the first case among construction site workers

${ }^{\mathrm{b}}$ Constructions sites costing ten million Singapore dollars and above were classified as large scale projects 

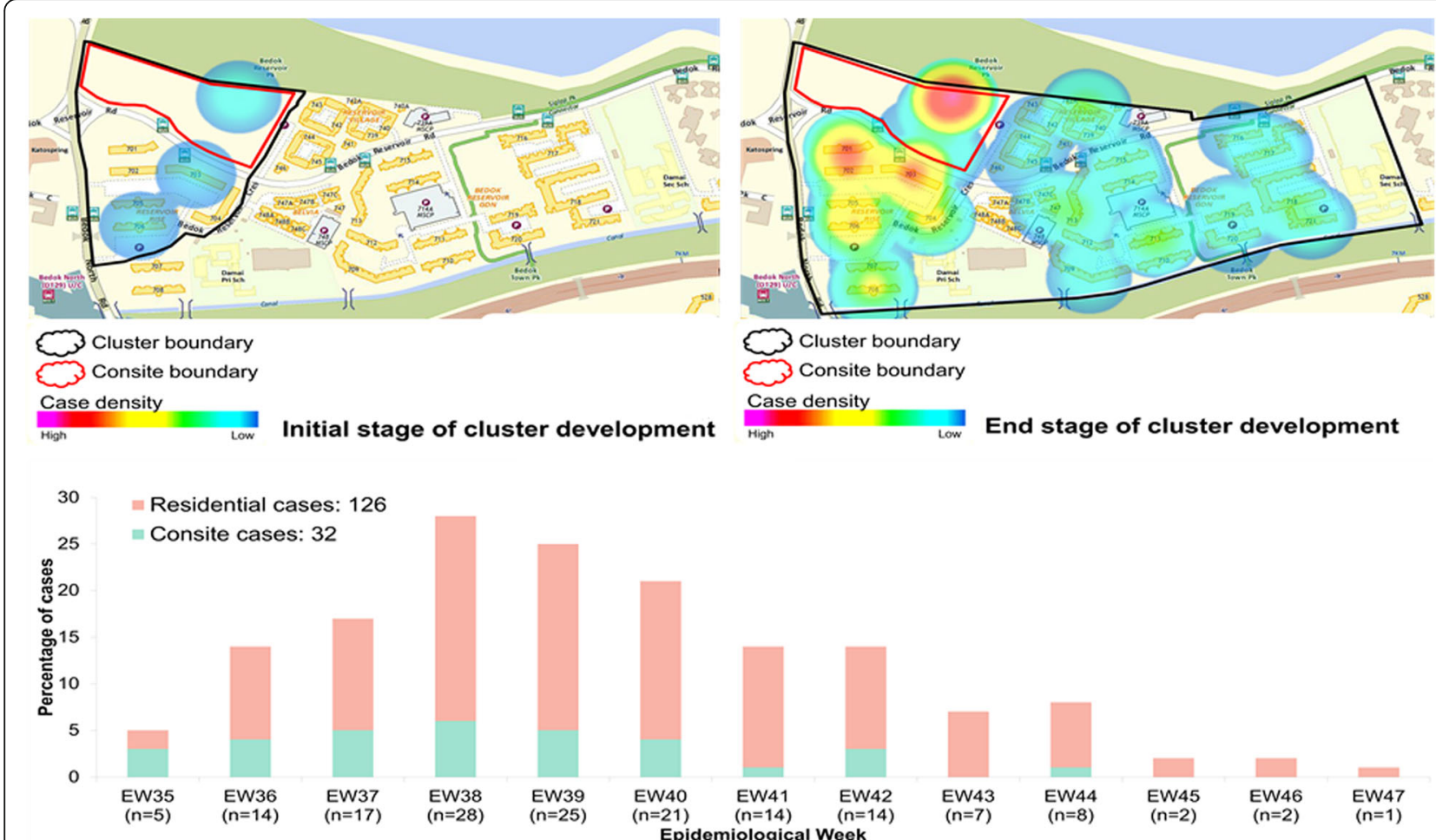

Fig. 1 Spatial case density map and temporal distribution of reported dengue cases in Bedok Reservoir Road cluster. The size of the cluster is 0.39 km². Cluster boundary is shown in black and the construction site boundary in red. The spatial fluctuations of case density during the initial and end stages of the cluster are shown in different shades of colour as per the legend. The graph below the maps shows the weekly distribution of cases among residents and construction site workers. Consite $=$ construction site

(unpublished data, Environmental Health Institute). Therefore, repeated introduction of the same variants from other distant sites cannot be completely ruled out.

\section{Discussion}

The overall case burden of construction site-associated clusters was significantly higher than that of remaining clusters and the probability of construction site-associated clusters expanding into major clusters was significantly high. These findings demonstrated the potential impact of construction sites on dengue transmission, especially when spill-over transmission takes place in surrounding residential areas, where the population may have relatively low herd immunity $[6,16]$. However, the ultimate size of clusters was not dependent on the size of the construction project or whether the cases initially occurred within the construction sites. Our case studies highlighted the likelihood of virus exchange between the construction sites and the immediate periphery that subsequently resulted in further expansion of clusters. The high homogeneity of dominant virus populations among construction workers and residents over the active period of study site clusters indicated potentially a strong transmission link between construction sites and residential areas. The fact that a higher proportion of cases in our case studies was detected among residents than the construction workers suggested the possibility of a two-way virus exchange between construction sites and surrounding areas once the transmission established.

These observations implied that the expansion of clusters was not purely attributable to cases in construction sites, but also due to the external neighbouring environment being conducive for virus transmission. Because the workers move around in the neighbourhood and mosquitoes could migrate between the construction sites and the external periphery, it is difficult to cease new cases until the transmission is interrupted in both environments. Nevertheless, as the workers are often highly congregated in construction sites, the disease tends to spread faster among workers than the residents. It has previously been reported that due to poor house-keeping, construction sites tend to create an environment conducive for mosquito breeding [17]. In the presence of suitable tropical climatic factors [18], coupled with the dynamic nature of construction sites, breeding habitats can be easily established and often be overlooked .

National Environment Agency (NEA)'s inspection statistics show that construction sites have the propensity of breeding mosquitoes with high larval density (Additional file 1: Figure S1 and Additional file 2: Table S1). As such, 

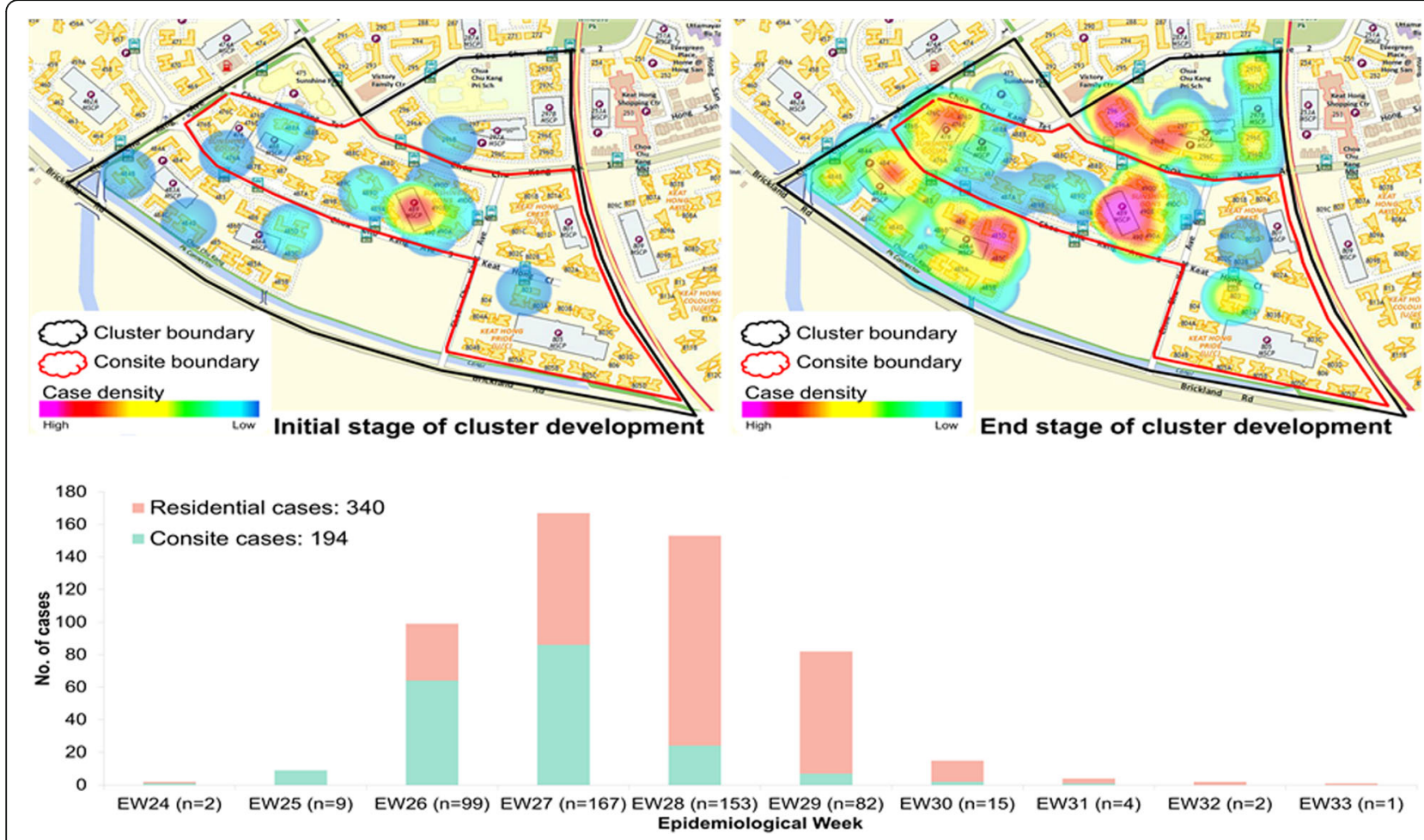

Fig. 2 Spatial case density map and temporal distribution of reported dengue cases Choa Chu Kang cluster. The size of the cluster is $0.46 \mathrm{~km}{ }^{2}$. Cluster boundary is shown in black and the construction site boundary in red. The spatial fluctuations of case density during the initial and end stages of the cluster are shown in different shades of colour as per the legend. The graph below the maps shows the weekly distribution of cases among residents and construction site workers. Consite $=$ construction site

construction sites, where mosquito breeding is detected or have poor housekeeping face stringent penalties, including Stop Work Orders and prosecution in Court. In addition, NEA has introduced additional measures since 2015 to enhance dengue control efforts in construction sites:

a. formation of dedicated teams for the inspection of construction sites for mosquito breeding. The inspection frequency at large construction sites has been stepped up from quarterly to monthly.

b. publishing the list of Stop Work Order sites to serve as a deterrent to contractors to maintain good housekeeping. The publication will also encourage contractors to promptly take actions to remove conditions favourable for mosquito breeding and to limit the potential of secondary infections in the area.

c. temperature screening of workers at construction sites in areas of dengue transmission to enable early case detection, application of insect repellents on both dengue-infected and healthy workers and quarantining infected workers under bed nets or in air-conditioned sick bays to prevent further transmission.
NEA has also implemented the Environmental Control Officers Scheme to mandate companies to take up the responsibility of minimizing vector breeding at large construction sites and protecting the staff from acquiring vector-borne diseases. The role of Environmental Control Officers is critical in preventing the initiation of new clusters.

One of the limitations of our study is the non-availability of entomological data to characterise mosquito density fluctuations at construction sites during different construction phases to highlight specific risk factor(s) for more targeted vector control operations. Without entomological data, it is difficult to pinpoint whether the relatively high transmission intensity of DENV in construction-site associated clusters is due to the abundance of vectors. Nevertheless, our data on higher premise index and more breeding habitats may be used as a proxy for higher vector abundance in construction sites than residential premises. On the other hand, high transmission could be driven by a continual source of viruses introduced into these sites through the labour-force as shown in our findings. The successful establishment of transmission is also determined by the immunological status of construction workers. Therefore, the source of transmission in construction sites seems to be 

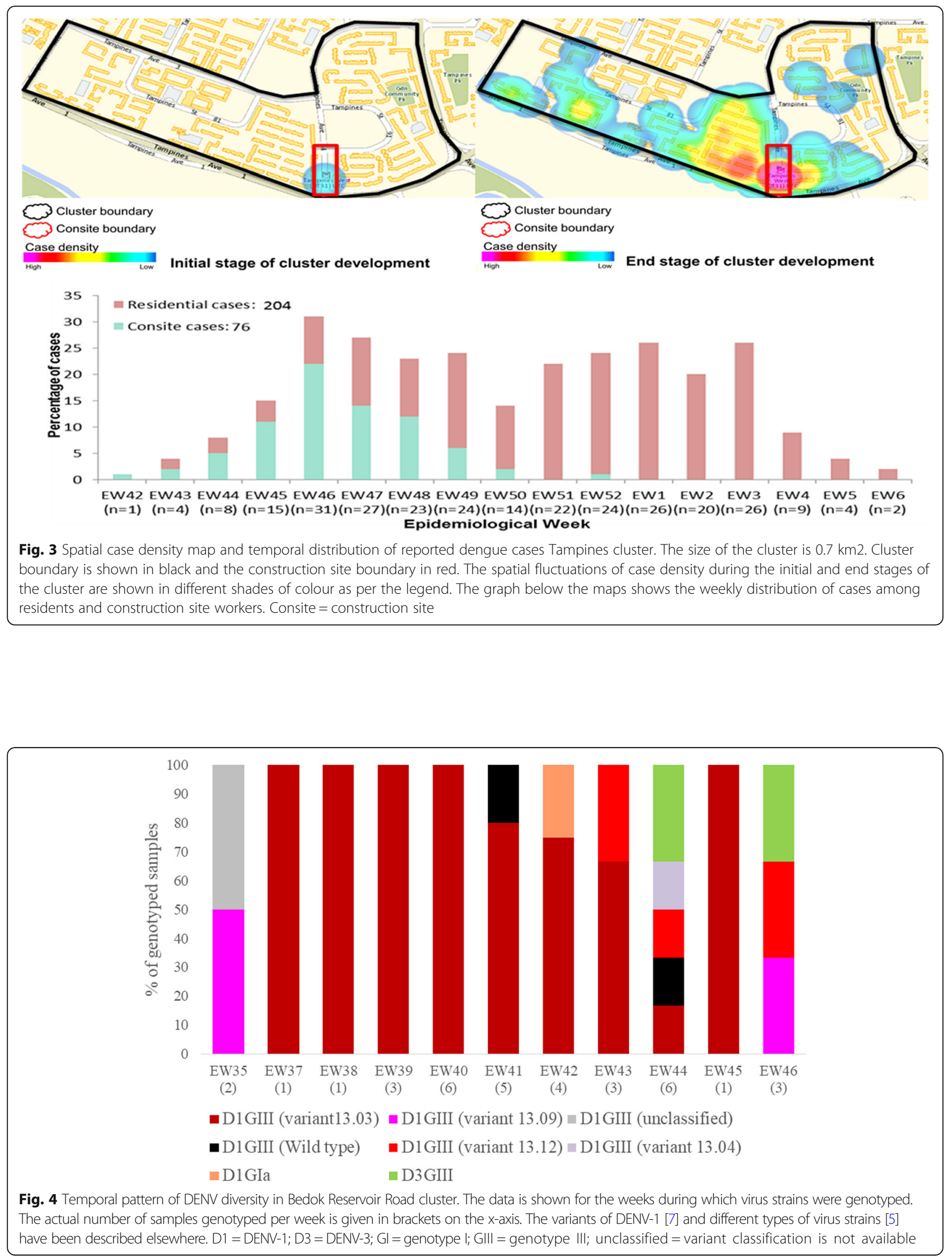


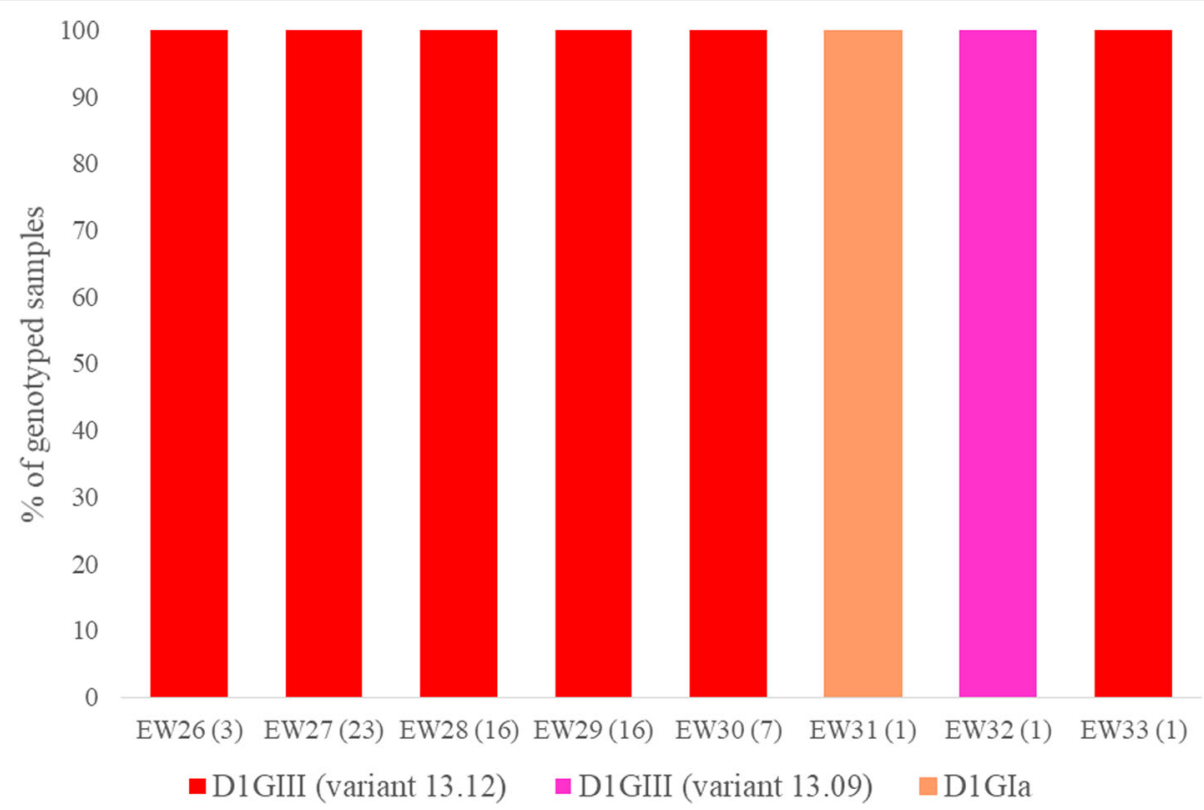

Fig. 5 Temporal pattern of DENV diversity in Choa Chu Kang cluster. The data is shown for the weeks during which virus strains were genotyped. The actual number of samples genotyped per week is given in brackets on the $x$-axis. The variants of DENV-1 [7] and different types of virus strains [5] have been described elsewhere. D1 = DENV-1; Gl = genotype I; GIII = genotype III

multi-factorial and opportunistic. Another limitation is the lack of information on the actual size of individual construction sites. We investigated whether the size of the construction sites impacts on the final size of clusters by using the estimated cost of the projects as a proxy. However, the data was not available for all construction sites during the study period. Moreover, the estimated project cost not only depends on the land area, but also on the land value that varies based on the location.

\section{Conclusions}

The role of construction sites as an important driver of dengue transmission cannot be underestimated. Despite

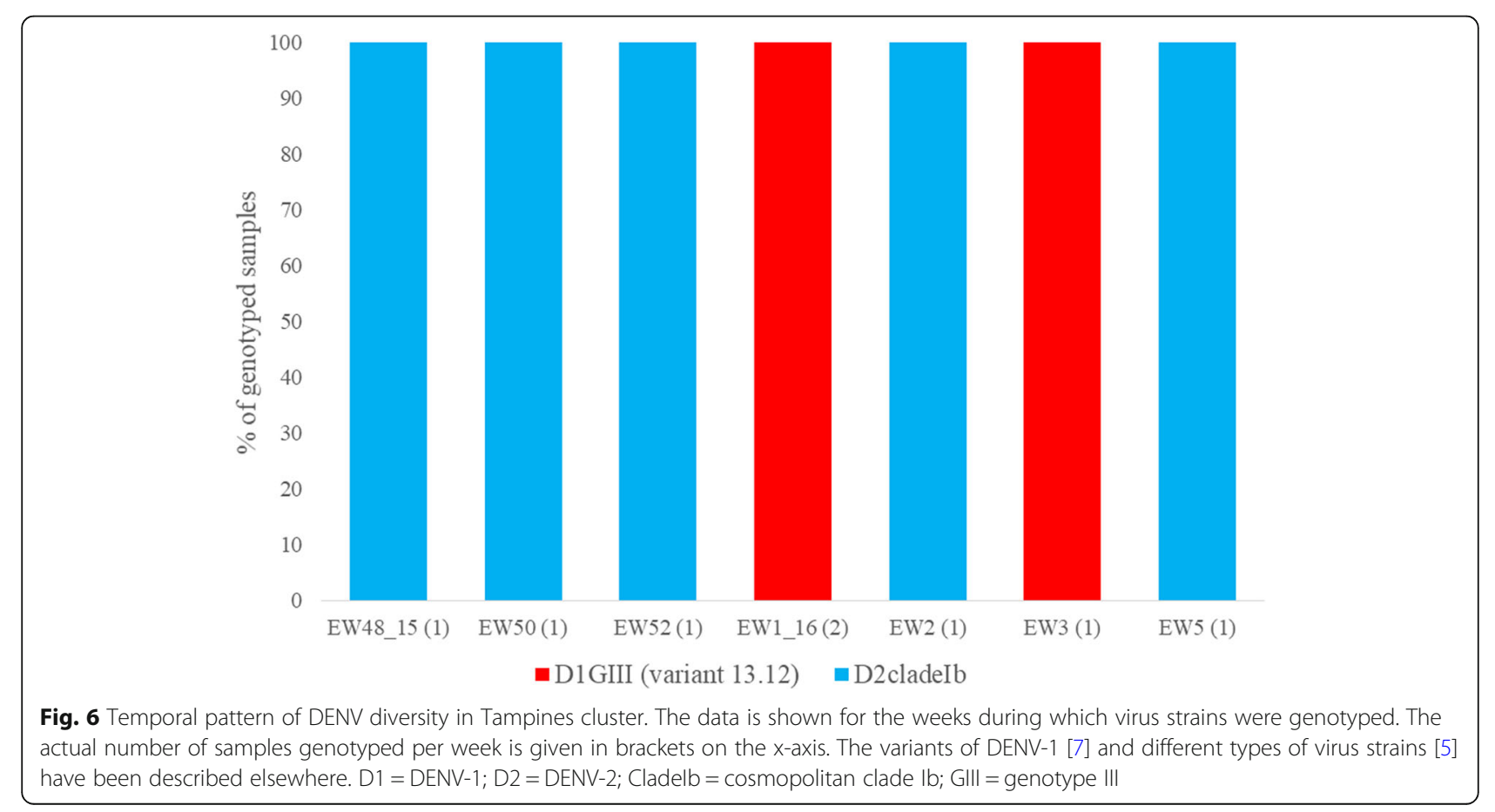


an intensive island-wide vector control program in Singapore, vector surveillance in construction sites is still a challenge given the large number of construction activities at any one time. The findings emphasise that dengue control measures of construction site-associated clusters should not be limited to the sites per se, but to stretch out to a "buffer-zone" in the surrounding residential areas to minimize sustained virus transmission. Further studies are warranted to characterise the mosquito breeding and population density fluctuations at sites during different phases of the construction work. As many dengue endemic countries in Asia and South/Central America have emerging economies and are undergoing rapid infrastructure upgrading, our findings have important implications for the policy planning and control of dengue in an era of rapid urbanisation.

\section{Additional files}

Additional file 1: Figure S1. Aedes Premises Index of government housing (HDB) flats, private apartments, landed properties, construction sites and dormitories (2013-2016). Premises Index is defined as the number of inspected premises found with Aedes breeding out of 100 inspected premises. (TIF $14 \mathrm{~kb}$ )

Additional file 2: Table S1. Mosquito breeding habitats and larvae counts detected in construction sites and residential premises from 2013 to 2016 (DOCX $12 \mathrm{~kb})$

\section{Abbreviations}

BR: Bedok Reservoir Road; CCK: Choa Chu Kang; DENV: Dengue virus; E: Envelope gene; EW: Epidemiological week; NEA: National Envioronment Agency; OR: Odds ratio

\section{Acknowledgements}

The authors acknowledge colleagues at the Environmental Health Institute, especially Ms. Hui-Leng Quek and Dr. Christina Liew and the Environmental Public Health Operations of the National Environment Agency, Singapore for their assistance in data collection and technical discussions.

\section{Funding}

The study was funded by the NEA, Singapore. The funding sources of this study had no role in the study design, data collection, data analysis, data interpretation, writing of the report, or in the decision to submit the paper for publication.

\section{Availability of data and materials}

All data generated or analysed during this study are included in this published article and its additional files. Envelope gene sequences generated during the present study were deposited in the GenBank (https:/www.ncbi.nlm.nih.gov/ genbank/) database (Accession no. MH428103 - MH428202 and MH428203 $\mathrm{MH} 428213)$.

\section{Authors' contributions}

LCN and GY conceptualized and designed the study. SL, CSC, CST, HCH, JR and $\mathrm{CK}$ were involved in the data collection, generation, processing and analyses. SL, CSC, CST and HCH drafted the manuscript. JR, CK, HCH, LCN and $\mathrm{GY}$ revised the manuscript critically for important intellectual content. All authors are accountable for every aspect of the work, especially the accuracy and integrity, and have read and approved the manuscript for publication.

\section{Ethics approval and consent to participate}

All sera used to generate $E$ gene sequences described in the present study were obtained from patients after obtaining the written informed consent. The use of consented patient sera for the genotyping purposes was approved by the Institutional Review Board of National Environment Agency, Singapore (IRB 003.1). The remaining data presented in the study was collected as part of the surveillance activities under the dengue control programme in Singapore.

Consent for publication

Not applicable

\section{Competing interests}

The authors declare that they have no competing interests.

\section{Publisher's Note}

Springer Nature remains neutral with regard to jurisdictional claims in published maps and institutional affiliations.

\section{Author details}

${ }^{1}$ Environmental Health Institute, 11, Biopolis Way, \#06-05-08, Singapore 138667, Singapore. 'Environmental Public Health Operations Department, National Environment Agency, 40, Scotts Road, \#13-00, Singapore 228231, Singapore. ${ }^{3}$ School of Biological Sciences, Nanyang Technological University, 60 Nanyang Drive, Singapore 637551, Singapore.

Received: 31 October 2017 Accepted: 3 August 2018

Published online: 08 August 2018

\section{References}

1. Murray NEA, Quam MB, Wilder-Smith A. Epidemiology of dengue: past, present and future prospects. Clin Epidemiol. 2013;5:299.

2. Guo C, Zhou Z, Wen Z, Liu Y, Zeng C, Xiao D, Ou M, Han Y, Huang S, Liu D, et al. Global epidemiology of dengue outbreaks in 1990-2015: a systematic review and meta-analysis. Front Cell Infect Microbiol. 2017;7:317.

3. Koh BK, Ng LC, Kita Y, Tang CS, Ang LW, Wong KY, James L, Goh KT. The 2005 Dengue epidemic in Singapore: epidemiology, prevention and control. Ann Acad Med Singapore. 2008;37(7):538-45.

4. Lee-Ching Ng HKT, Tan L-K, Chong C-S, Ho D. Evolving Dengue Control Programme in Singapore. Epidemiological News Bulletin. 2016;42:16.

5. Hapuarachchi HC, Koo C, Rajarethinam J, Chong CS, Lin C, Yap G, Liu L, Lai $\mathrm{YL}$, Ooi PL, Cutter J, et al. Epidemic resurgence of dengue fever in Singapore in 2013-2014: a virological and entomological perspective. BMC Infect Dis. 2016;16:300.

6. Low SL, Lam S, Wong WY, Teo D, Ng LC, Tan LK. Dengue seroprevalence of healthy adults in Singapore: serosurvey among blood donors, 2009. Am J Trop Med Hyg. 2015;93(1):40-5.

7. Hapuarachchi HC, Koo C, Kek R, Xu H, Lai YL, Liu L, Kok SY, Shi Y, Chuen RL, Lee KS, et al. Intra-epidemic evolutionary dynamics of a dengue virus type 1 population reveal mutant spectra that correlate with disease transmission. Sci Rep. 2016;6:22592.

8. Banu S, Hu W, Hurst C, Tong S. Dengue transmission in the Asia-Pacific region: impact of climate change and socio-environmental factors. Trop Med Int Health. 2011;16(5):598-607.

9. Hammond SN, Gordon AL, Lugo Edel C, Moreno G, Kuan GM, Lopez MM, Lopez JD, Delgado MA, Valle SI, Espinoza PM, et al. Characterization of Aedes aegypti (Diptera: Culcidae) production sites in urban Nicaragua. J Med Entomol. 2007;44(5):851-60.

10. Morrison AC, Gray K, Getis A, Astete H, Sihuincha M, Focks D, Watts D, Stancil JD, Olson JG, Blair P, et al. Temporal and geographic patterns of Aedes aegypti (Diptera: Culicidae) production in Iquitos, Peru. J Med Entomol. 2004;41(6):1123-42.

11. Ministry of Health: Communicable Diseases Surveillance in Singapore 2013.; 2014.

12. Ministry of Health: Communicable Diseases Surveillance in Singapore 2014.; 2015

13. Ministry of Health: Communicable Diseases Surveillance in Singapore 2015:; 2016.

14. Altman DG. Practical statistics for medical research. London: Chapman \& Hall/CRC; 1990.

15. Koo C, Nasir A, Hapuarachchi HC, Lee KS, Hasan Z, Ng LC, Khan E. Evolution and heterogeneity of multiple serotypes of dengue virus in Pakistan, 2006-2011. Virol J. 2013;10:275.

16. Yew YW, Ye T, Ang LW, Ng LC, Yap G, James L, Chew SK, Goh KT. Seroepidemiology of dengue virus infection among adults in Singapore. Ann Acad Med Singap. 2009;38(8):667-75. 
17. Van Der Hoek W, Konradsen F, Amerasinghe PH, Perera D, Piyaratne MK, Amerasinghe FP. Towards a risk map of malaria for Sri Lanka: the importance of house location relative to vector breeding sites. Int J Epidemiol. 2003:32(2):280-5.

18. Burattini MN, Chen M, Chow A, Coutinho FA, Goh KT, Lopez LF, Ma S, Massad E. Modelling the control strategies against dengue in Singapore. Epidemiol Infect. 2008;136(3):309-19.

Ready to submit your research? Choose BMC and benefit from:

- fast, convenient online submission

- thorough peer review by experienced researchers in your field

- rapid publication on acceptance

- support for research data, including large and complex data types

- gold Open Access which fosters wider collaboration and increased citations

- maximum visibility for your research: over $100 \mathrm{M}$ website views per year

At $\mathrm{BMC}$, research is always in progress.

Learn more biomedcentral.com/submissions 